\title{
In-Cell Matrix-Assisted Laser Desorption-Ionization Fourier Transform Ion Cyclotron Resonance Mass Spectrometry
}

\author{
M. Knobeler and K. P. Wanczek \\ Institut für Anorganische und Physikalische Chemie, Universität Bremen, Bremen, Germany
}

\begin{abstract}
A new internal matrix-assisted laser desorption-ionization (MALDI) Fourier transform ion cyclotron resonance-mass spectrometry (FTICR-MS) method is introduced. The target is directly positioned at one trapping electrode of a single cylindrical ion cyclotron resonance (ICR) cell and becomes a part of it. The ionization occurs inside the ICR cell in contrast to external or near-cell MALDI-FTICR-MS techniques. Very efficient trapping and mass resolving power better than unit resolution of singly charged peptides and proteins ions up to 2000 $\mathrm{u}$ is possible by using only basic FTICR-MS techniques. The sole application of a pulsed retarding potential increases the mass range to $6000 \mathrm{u}$. No collisional cooling and quadrupolar excitation was done. Sensitivities below $1 \mathrm{fmol}$, and ion storage times of more than $15 \mathrm{~s}$ are shown. High resolving powers of 16,000 and 56,000 are obtained on bovine insulin (5.7 ku) and gramicidin D (1.9 ku), respectively. (J Am Soc Mass Spectrom 1996, 7, 1026-1033)
\end{abstract}

$\mathrm{T}$ The interest in coupling matrix-assisted laser desorption ionization (MALDI) [1,2] and Fourier transform ion cyclotron resonance mass spectrometry (FTI CR-MS) [3-6] increased in recent years to take advantage of the features of both MALDI and FTI CR-MS $[7,8]$. Unfortunately, the high kinetic energies and the high mass-to-charge ratios $(\mathrm{m} / \mathrm{z})$ of the MALDI-produced ions are disadvantageous for the ion cyclotron resonance (ICR) technique $[9,10]$, which requires efficient trapping of low energy ions for high mass resolution. The potential to trap ions is limited by the initial velocity and by the mass-to-charge ratio, that is, by the initial kinetic energy. Other factors, such as the electrostatic and magnetic fields, the cell dimensions, space charge effects, and the background pressure, limit the ion storage [11-16].

For the last several years, different new ICR techniques have been developed to trap heavy ions with energies higher than thermal energy and to cool the trapped ions to lower energies. Most of these techniques were employed successfully for MALDI-FTICRMS experiments. Moreover, additional methods were developed especially for MALDI-FTICR-MS. Several groups introduced different couplings of MALDI and FTICR-MS that partially used elaborate new ICR techniques. Both external MALDI with an electrostatic lens system [17] or with a quadrupole ion guide [18-20] and MALDI in close proximity to the cell [21-31] were

Address reprint request to Dr. K. P. Wanczek, Haferwendeiz, Universität Bremen, D-28334 Bremen, Germany. introduced. The target is positioned some millimeters or centimeters outside the ICR cell in the published experiments with a dual cell [21-29], a "waiting room" for collisional cooling [30], a cylindrical closed cell [30], or a cylindrical open cell [31]. Castoro and co-workers [21-23] trapped ions generated in front of a dual cell with a pulsed deceleration potential as a modification of the pulsed trapping used by Kofel et al. [32,33], McIver et al. [18, 19], and Hofstadler and Laude [34]. Castoro et al. [24] also employed a "screened" cell as proposed by Wang and Marshall [35] and Bamberg and Wanczek [36]. Solouki et al. [37] achieved low resolution spectra of a singly charged mass of $157 \mathrm{ku}$ by using a wire ion guide inside the ICR cell for better focusing. Mclver and co-workers [18-20] trapped externally generated ions by a pulsed trapping field and a pulsed inlet of collision gas. Resolution of 90,000 on bovine insulin $(5.7 \mathrm{ku})$ and high accuracy were obtained. Huang et al. [26] used quadrupolar excitation for axialization $[13,38,39]$ inside the source cell and demonstrated the possibility of $\mathrm{MS}^{4}$ experiments of MALDI-generated ions. Pastor et al. [25] reached a resolution of 92,000 on bovine insulin after quadrupolar excitation in the source cell and transfer of the ions into the analyzer cell. Pasa-Tolic et al. [27] showed a resolution of $1,500,000$ on $m / z=1 \mathrm{ku}$ after correction for frequency drift with the same method. Resolving power of $1,500,000$ at $m / z=1.6 \mathrm{ku}$ was achieved by Sheng et al. [29]. Aside from the modified ICR techniques, the use of fructose as a co-matrix was introduced to minimize metastable decay of the quasimolecular ions $[22,23]$. 
In this article we introduce a new in-cell MALDIFTICR-MS method for ionization inside the cell. A cylindrical cell is used with grid trapping electrodes to guarantee the lowest possible pressure inside the cell achievable by the vacuum system. The ions are produced by MALDI at the target that is a part of the front trapping electrode and therefore at an inner mechanical and electrical boundary of the ICR cell. Thus the ionization occurs directly inside the ICR cell. The trapping grid opposite to the target can be pulsed to higher voltages up to $50 \mathrm{~V}$. No quadrupolar excitation and typically no pulsed inlet of collision gas were employed for the presented results. Therefore the energy of the axial motion could be influenced only by the retarding potential. The energy of the radial motions, that is, the reduced cyclotron and the magnetron motion, was influenced only by minor effects before the dipolar excitation for image current detection, for example, the radial component of the retarding potential and space-charge effects.

The results presented characterize the potentialities of the introduced in-cell MALDI-FTICR-MS technique that uses only the basic ICR sequence Q-d-I-d-E-d-D and a pulsed retarding potential $(Q=$ ion quenching, $\mathrm{d}=$ delay, $\mathrm{I}=$ ionization, $\mathrm{E}=$ dipolar excitation, $\mathrm{D}=$ image current detection). This method is very simple because it does not employ complicated techniques of cooling by collision gas or by quadrupolar excitation, and it is sufficient to resolve the isotopes of bovine insulin $(16,000$ resolution at $m / z=5.7 \mathrm{ku})$ after a single scan. A sensitivity below $1 \mathrm{fmol}$ is demonstrated.

\section{Experimental}

\section{FTICR-MS Instrumentation}

The experiments were performed with a modified prototype Bruker Spectrospin CMS-47 Fourier transform ion cyclotron resonance mass spectrometer (BrukerFranzen Analytik GmbH, Bremen, Germany) equipped with a 7-T superconducting magnet with a horizontal room temperature 89-mm-diameter bore (Oxford Instruments, Oxford, UK). The preamplifier was removed from the room temperature bore and connected with the feed-through flange for the ICR cell by shielded lines. The homemade movable vacuum system is equipped with a $600-\mathrm{L} / \mathrm{s}$ oil diffusion pump with a nitrogen cryobaffle (VHS600, Varian Vacuum Division, Turin, Italy) and an adjustable piezoelectric gas leak valve (MV-112, Maxtek Inc., Torrance, CA). A three-stage push rod lock allows the vacuum system to be pumped down to a pressure of $1 \times 10^{-8} \mathrm{mbar}$ in less than $3 \mathrm{~min}$ after introduction of a solid sample at the probe tip into the vacuum system [40] (see Figure 1).

A homemade cylindrical ICR cell of 21-mm radius and $60-\mathrm{mm}$ length was employed. The trapping electrode at each end is a grid with a centered 3-mm-diameter hole. The cylinder electrode consists of eight seg-

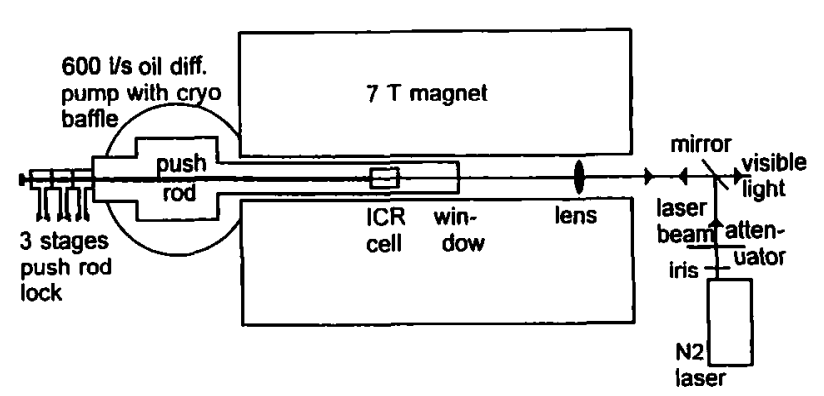

Figure 1. Diagram of the MALDI-FTICR-MS configuration. The position of the laser spot is controlled by the fluorescence of the matrix visible through the semipermeable mirror. The lens is located outside the vacuum for variation of laser spot size.

ments (sideplates) of equal size. Each two adjacent side plates are connected electrically for standard use. The target at the probe tip can be positioned directly at the front trapping grid of the ICR cell. The center of the target is slightly misaligned against the center of the front grid as shown in Figure 2. The voltage supplies of the front trapping grid and the target are separated and can be connected outside of the vacuum system. In case of electrical connection, as for all presented spectra the target becomes a part of the front trapping electrode. A small distance of some hundred micrometers isolates the grid and the target if different voltages are required.

\section{MALDI Instrumentation}

A pulsed 337.1-nm nitrogen laser (VSL-337ND, Laser Science Inc., Newton, MA) with $200 \mu \mathrm{J}$ per 3-ns pulse was used. The laser beam passes an attenuator, is reflected by $90^{\circ}$ by a semipermeable mirror, and focused by a 1 -in.-diameter lens of $50-\mathrm{cm}$ focal length. The beam enters the vacuum through a fused silica window and crosses the ICR cell through the orifices in

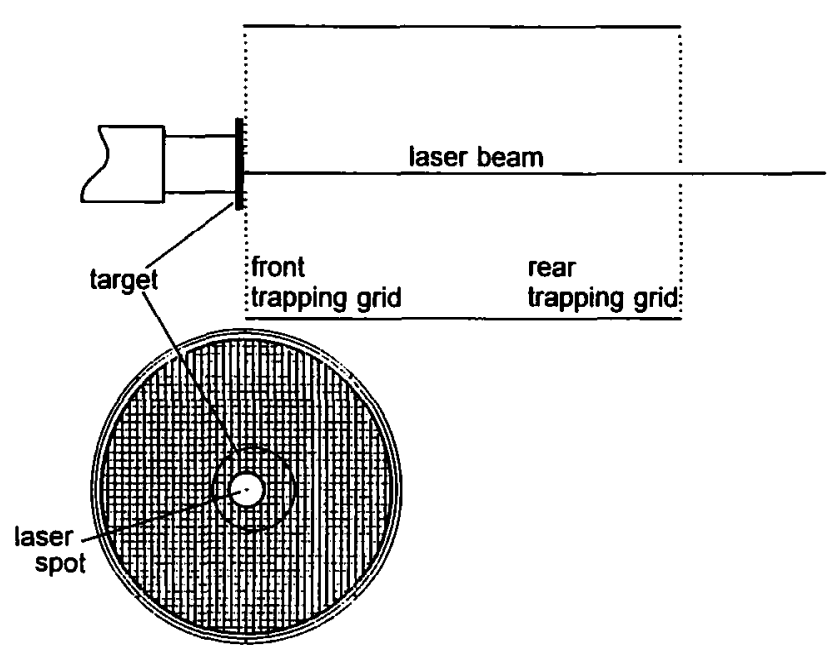

Figure 2. Diagram of the ICR cell with the target as a part of the front trapping electrode. Because the center of the target is slightly misaligned, the laser hits different spots by manual rotation of the sample probe. 
the trapping grids to hit the target at the front trapping grid. The incidence angle at the target is $0^{\circ}$ and the focused beam diameter at the target is $\sim 200-500 \mu \mathrm{m}$. The lens positioned inside the room temperature bore of the magnet but outside the vacuum enables an easy variation of the focused beam diameter at the target.

The fluorescence of 2,5-dihydroxybenzoic acid applied to the target, a small halogen lamp inside the vacuum system, and a mirror that is permeable for visible light allow the optical alignment of the laser beam on the target at the symmetry center of the front trapping grid. The symmetry center of the trapping grid is not identical to the rotation center of the target. Once the laser beam is aligned, the laser hits the target at the center of the trapping grid and the push rod has to be rotated only for different spots at the target to be hit.

\section{Sample Preparation}

The presented data all were achieved with $0.5-\mathrm{M} 2,5-$ dihydroxybenzoic acid (DHB; Fluka Chemie AG, Buchs, Switzerland) of a concentration between 0.5 and approximately $3 \mathrm{M}$ (saturated) in isopropanol-water 1:1 as matrix with or without co-added $0.5-\mathrm{M}$ fructose. The concentrations of the samples (purchased from Fluka) were $10^{-4}-10^{-7} \mathrm{M}$. Amounts between 0.1 and 1 $\mu \mathrm{L}$ of the solutions were applied to the target by a microsyringe or $10-50 \mu \mathrm{L}$ were aerosprayed onto the target.

\section{Pulse Generation, Pulse Sequences, and Data Processing}

To analyze masses higher than $1000 \mathrm{u}$ the rear trapping grid was switched to a deceleration voltage of 5-50 V triggered simultaneously with the laser. The voltage was changed to the standard trapping potential of $0.3-3 \mathrm{~V}$ after $80-200 \mu \mathrm{s}$. No pulsed deceleration voltage was used for the result shown in Figure 3 . The voltages at the front trapping grid and at the target were fixed at $0.3-3 \mathrm{~V}$ during the experiments. All sequences, such as triggering, delaying, quenching, excitation, and image current detection, were performed with the standard technique of the CMS spectrometer except the supply of the rear trapping grid voltage. The pulsed voltage at the rear trapping grid is generated with a sweep generator (Model 164, Wavetek, San Diego, CA) triggered simultaneously with the laser by the ionization pulse of the spectrometer and amplified with a homemade pulse amplifier. The standard pulse and delay sequence of the software were used if one laser shot per scan and no pulsed gas inlet was employed (all presented spectra except Figure 5). A laser pre-shot before ion quenching and/or a pulsed gas inlet were controlled by a short pulse program written with the software.

Single scans were acquired or 10-100 signals added. The data points were apodized by Gaussian functions

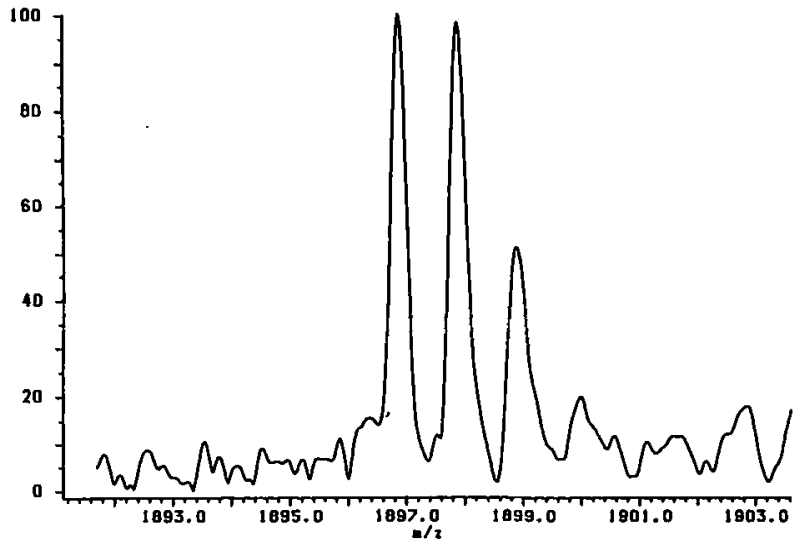

Figure 3. Spectrum with a mass resolution of 5000 of gramicidin $\mathrm{D}$ achieved with fixed voltages of $1 \mathrm{~V}$ at the front trapping grid and target and $1.5 \mathrm{~V}$ at the rear trapping grid. Signals of 16 scans were added prior to transformation. The analyte-DHBfructose ratio was 1:10,000:0. The trapping without pulsed retarding potential was successful at masses up to $2 \mathrm{ku}$.

(Figure 3a, 7-9), two times zero-filled (i.e., the number of data points was increased by a factor of 4 ), and Fourier transformed to magnitude spectra except for Figure 8 , which shows an absorption spectrum after phase correction. No calibration was done because these initial experiments were achieved under different conditions such as deceleration and trapping potentials.

\section{Results and Discussion}

\section{Desorption/Ionization}

The effect of co-added fructose $[22,23]$ was not unambiguously reproducible, although the laser power density was adjusted carefully by attenuation or minor defocusing to operate at the desorption threshold for MALDI [23]. A consecutive loss of $17 \mathrm{u}$ but no further fragmentation by metastable decay of bovine insulin was observed by using a matrix with and without co-added fructose; the relative abundance of the protonated molecular ion was higher in most experiments with co-added fructose. The highest resolution on bovine insulin was obtained with a matrix that contained fructose. Nevertheless, the resolution was generally independent of the matrix.

The spraying of the solution as aerosols onto the target yields more reliable and reproducible results than the direct application with a microsyringe. Unfortunately the quantity needed for spraying is higher than for direct application, although the quantity consumed per laser shot may be the same. The ionization was not successful for some experiments when the delay between two complete scans and thus between two laser shots was longer than 100-500 ms (during signal accumulation or tuning with single scans). This phenomenon probably depended on the target preparation, but it occurred sometimes after spraying and 
sometimes after direct application. The ionization could be improved greatly by a laser pre-shot in such cases. The laser was triggered the first time before ion quenching and than triggered a second time with ion trapping. The delay between the pre-shot and the second shot was 50-200 ms. No ions were trapped after the first shot because it occurred before ion quenching. A possible interpretation may be the warm up of the matrix by the first laser shot for better ionization by the second shot. A necessary warming up of the laser is another explanation as well. However, all presented spectra were achieved without a laser pre-shot except the spectrum in Figure 5.

\section{Ion Trapping Without Pulsed Deceleration}

Figure 3 shows a spectrum of gramicidin $\mathrm{D}(M=$ $1.9 \mathrm{ku}$ ) achieved with fixed voltages of $1 \mathrm{~V}$ at the target and the front trapping grid and $1.5 \mathrm{~V}$ at the rear trapping grid. The matrix was DHB without co-added compounds and the background pressure was $5 \times$ $10^{-8}$ mbar. Note that neither were both trapping electrodes pulsed to ground potential as by Solouki and Russell [30] nor was the rear trapping electrode pulsed to higher voltage as by Castoro et al. [21]. These experiments are comparable with the initial MALDI-FTICR-MS experiments of Hettich and Buchanan [10]. The resolution achieved here is much higher than the presented results of Hettich and Buchanan, although they used a 3-T magnet. Our high resolution experiments without pulsed trapping voltages were successful at masses up to $2 \mathrm{ku}$.

Different voltages at the target and the front trapping grid, especially a lower potential at the target for retarding, had only minor or no effects. The best results were obtained with a slightly asymmetrical potential in the axial direction caused by different voltages at the trapping grids. The reason for this is not obvious. The asymmetric trapping potential shifts the center of the ion cloud that possibly corrects the nonideal position of the $6-\mathrm{cm}$-long cell in the magnetic field.

\section{Ion Trapping with Pulsed Deceleration}

The operation with a pulsed retarding potential yielded better results at masses higher than $1 \mathrm{ku}$ and was necessary to trap the quasi-molecular ions of bovine insulin $(5.7 \mathrm{ku})$. Whereas the voltage at the rear trapping grid can be raised to $50 \mathrm{~V}$, the target and the front trapping grid need not be set to ground potential during deceleration as used by other authors [21,26], because the applied trapping voltage can be compensated by an appropriate higher potential at the rear trapping grid. Experiments with a lower or higher voltage at the target than at the front trapping grid showed that any improvement by this application can be obtained by a higher or lower retarding voltage as well.
Ions with kinetic energies up to $50 \mathrm{eV}$ can be trapped theoretically by the increased retarding potential of $50 \mathrm{~V}$. By this, there is no limitation to retardation of ions with an initial axial velocity up to $980 \mathrm{~m} / \mathrm{s}$ and masses up to $10 \mathrm{ku}$. Unfortunately, bovine insulin (5.7 $\mathrm{ku})$ is currently the highest detected mass. The reason for this failure can be the imperfect pulse shape at higher voltages or unoptimized analyte-matrix ratios.

The retarding voltage, the retarding time (i.e., the length of the pulse for the retarding voltage), and the trapping voltages at the front and rear trapping grids are important parameters for ion trapping and storage. The parameters that are adequate for sufficient ion trapping for low resolution experiments expand over a wide range. The conditions for ion storage times longer than a few hundred milliseconds and for high resolution experiments were much more sensitive, especially for higher masses such as bovine insulin.

The exact and independent control of the trapping voltages at the front trapping grid (which includes the target) and the rear trapping grid was essential. The longest ion storage time was possible only with an axial asymmetrical trapping field that is $1.2-3$ times higher at the rear trapping grid than at the front trapping grid (see foregoing discussion). Whereas the best conditions for ion storage and high mass resolution are low trapping potentials (especially at higher masses), the highest signal-to-noise ratios at low mass resolution were obtained at higher trapping voltages.

The values of the retarding voltage and the retarding time were important but less critical. The retarding time could be varied over a range of some $10 \mu \mathrm{s}$ (at $100-200 \mu \mathrm{s}$ optimum) at a fixed voltage without significant change in the spectra. Moreover, lower retarding voltages could be compensated by longer retarding times. However, the longest storage times were achieved with relatively low retarding potentials and medium retarding times.

\section{Ion Storage}

The low resolution spectra of bovine insulin shown in Figure 4 were obtained by a variable delay after trapping with a pulsed retarding potential. The retarding voltage of $30 \mathrm{~V}$ was maintained for $150 \mu \mathrm{s}$. The trapping voltages were 0.3 and $0.55 \mathrm{~V}$ at the front trapping grid (which includes the target) and at the rear trapping grid, respectively. The background pressure was $1 \times 10^{-8}$ mbar. A signal was detectable up to a delay of $30 \mathrm{~s}$ with a signal-to-noise ratio of 3 (most abundant peak). The most abundant peak is the ion $[\mathrm{M}+\mathrm{H}-$ $17]^{+}$that is independent of the storage time.

Experiments were carried out with permanent and pulsed gas inlets to prove the ion storage at different pressures. Figure 5 shows a spectrum of bovine insulin after a pulsed gas inlet of nitrogen. The pulsed valve was opened $100 \mathrm{~ms}$ before the ionization event to generate a pressure of $1 \times 10^{-4}$ mbar inside the ICR cell. The pressure was maintained for $5 \mathrm{~s}$ after ioniza- 


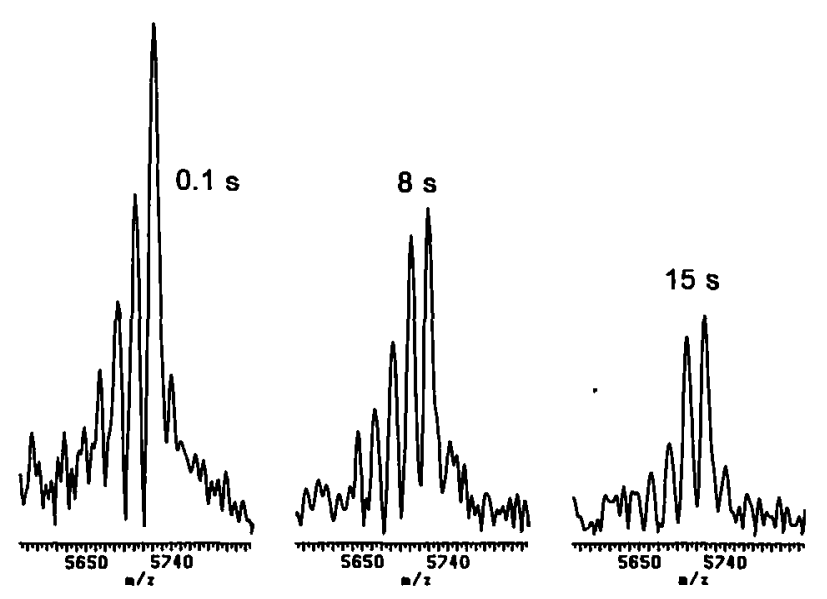

Figure 4. Spectra of bovine insulin (each 10 scans, analyteDHB-fructose ratio 1:5000:5000) obtained with a variable delay of $100 \mathrm{~ms}, 8 \mathrm{~s}$, and $15 \mathrm{~s}$ before dipolar excitation. A signal was detectable up to a delay of $30 \mathrm{~s}$. No quadrupolar excitation was used at a background pressure of $1 \times 10^{-8}$ mbar.

tion. The valve was closed and after an additional $2 \mathrm{~s}$ for pumping down to $1 \times 10^{-7}$ mbar, the ions were dipolarly excited and the image current was detected. Thus, the total storage time before dipolar excitation was $7 \mathrm{~s}$, which included a pressure of $1 \times 10^{-4} \mathrm{mbar}$ for at least $5 \mathrm{~s}$. Comparable results were obtained at a static pressure of $1 \times 10^{-4} \mathrm{mbar}$.

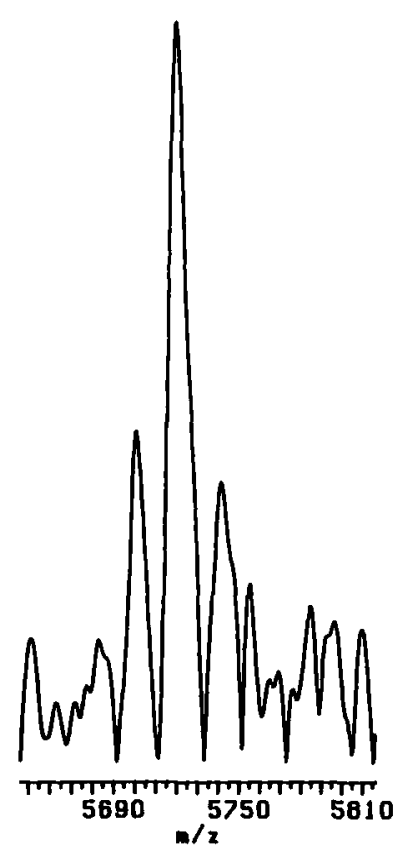

Figure 5. Spectrum (16 scans; analyte-DHB-fructose ratio $1: 10,000: 10,000)$ of bovine insulin obtained after a 7-s delay at increased pressure before dipolar excitation. A pressure of $1 \times$ $10^{-4}$ mbar was maintained for $5 \mathrm{~s}$ and pumped down to $1 \times$ $10^{-7}$ mbar during the additional $2 \mathrm{~s}$ before dipolar excitation.

\section{Resolution}

The spectra in Figure 6 demonstrate the ability of image current detection at different static pressures maintained by admitting argon through the continuously open adjustable pulsed valve. The ions were dipolarly excited after trapping with a retarding potential and a short storage time of $100 \mathrm{~ms}$. The obtained resolutions [full width at half peak height (FWHH)] are 60,350 , and 450 at a static pressure of $1 \times 10^{-3}$, $1 \times 10^{-4}$ and $1 \times 10^{-5}$ mbar, respectively. The capability of the ICR technique to achieve spectra of high mass compounds at a surprising low vacuum should be noted.

Whereas the lengths of the time domain signals and therefore the resolutions at the experiments of Figure 6 depend clearly on the pressure, a very carefully balanced set of the parameters was indispensible to further increase the resolution at lower pressures. Figure 7 shows a spectrum with baseline resolved isotopes (16K resolution) of bovine insulin at a pressure of $1 \times 10^{-8}$ mbar. Comparable results were achieved at $1 \times 10^{-7}$ mbar. Figure 8 shows a spectrum of gramicidin $\mathrm{D}$ with a resolution of $56 \mathrm{~K}$ obtained at $5 \times$ $10^{-8}$ mbar. The best resolutions were achieved only with a short delay of $50-500 \mathrm{~ms}$ after the ionization event and a low static pressure of $10^{-7}-10^{-8}$ mbar. Note that no mass calibration was done because these initial results were achieved at different conditions, such as trapping and retarding voltages. The relative peak heights of the isotopes at the high resolution spectra differ from the isotope patterns of the samples.

\section{Time Dependent Behavior of Stored Ions}

The results prove the ability to trap and to store in-cell MALDI-generated ions. The number of stored ions and therefore the signal-to-noise ratio depends on the pres-

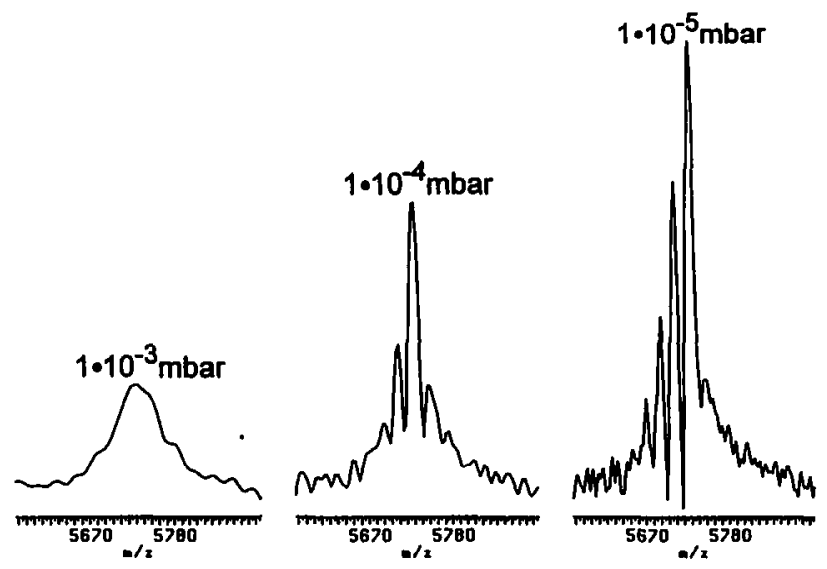

Figure 6. Spectra (each 10 scans, analyte-DHB-fructose ratio 1:30,000:0) of bovine insulin achieved at $1 \times 10^{-3}, 1 \times 10^{-4}$, and $1 \times 10^{-5}$-mbar static pressure. The mass resolution is 60,350 and 450 , respectively. A better tuning of the voltages was necessary to further increase resolution at lower pressures. Note the capability to obtain moderate spectra at surprisingly low vacuum. 


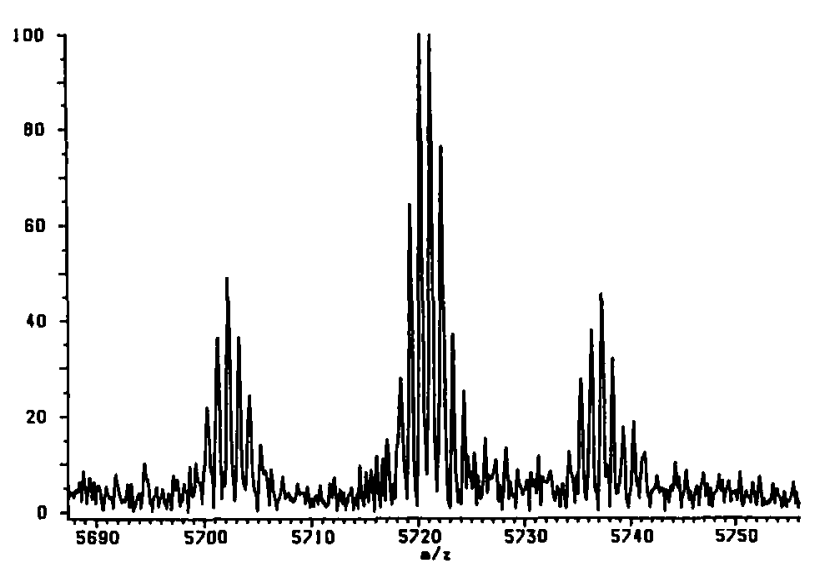

Figure 7. High resolution spectrum of bovine insulin obtained after a single scan experiment (analyte-DHB-fructose ratio 1:5000:5000). The isotopes are baseline resolved at a resolution of 16,000 (FWHH). The spectrum was achieved by $0.3 \mathrm{~V}$ at the front trapping grid (which included the target) and $30 \mathrm{~V}$ at the rear trapping grid during 150- $\mu$ s retarding time. Voltages of 0.30 and $0.55 \mathrm{~V}$ were applied at the front grid (which included the target) and rear grid, respectively, for ion storage during a delay of $100 \mathrm{~ms}$ before dipolar excitation and during the acquisition time of $1.5 \mathrm{~s}$.

sure and on the storage time (see Figures 5, 6, and 4, respectively). The longest transients and thus the highest resolutions were obtained only after relatively short storage times (Figures 7 and 8 ). This situation can be caused by collisions of the ions with neutrals of masses much lower than the mass of the molecular ion $[12,38]$. The radius of the cyclotron motion and the amplitude of the axial trapping motion decrease while the radius of the magnetron motion increases much slower by collisions.

Anderson and Laude [41] showed recently that storage of bovine insulin ions was possible for only 5$10 \mathrm{~ms}$ without quadrupolar excitation and for more than $10 \mathrm{~s}$ with application of quadrupolar excitation.

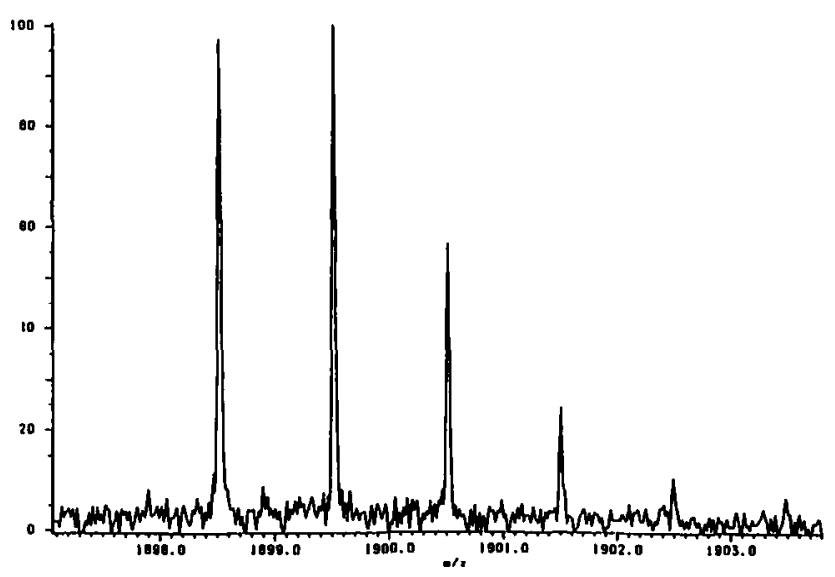

Figure 8. High resolution spectrum (100 scans; analyteDHB-fructose ratio 1:10,000:0) of gramicidin $D$ by using a pulsed retarding potential. Very carefully balanced voltages were required to achieve the resolving power of 56,000 . Resolutions of $10,000-15,000$ at masses up to $3 \mathrm{ku}$ were obtained without intensive tuning.
However, experiments by other authors $[18,19,42,43]$ were successful with ion cooling by collisions or even with remeasurements by collisions that used very long delays or pulsed increased pressure but no quadrupolar excitation. Speir et al. [44] showed that ion cooling without quadrupolar excitation depends much more on the experimental parameters than on cooling with quadrupolar excitation. The parameters of the experiments presented here were optimized for low pressures. No fine tuning for increased pressure was done. An optimization of the parameters for collisional cooling should be possible. Nevertheless, neither collisional cooling without nor with quadrupolar excitation was necessary to obtain spectra of masses up to $5.7 \mathrm{ku}$ with resolved isotopic peaks and a signal-to-noise ratio of about 10 after a single scan experiment as shown in Figure 7. Low resolution spectra also were achieved after relatively long ion storage as shown in Figure 4.

\section{Sensitivity}

Whereas the in-cell technique produces all ions inside the analyzer cell, no ions will be lost before they enter the cell as in the methods in which ions are generated outside the ICR cell or even outside the room temperature bore of the magnet. The radial motion and the aperture of the front trapping electrode have to be considered if the target is positioned near but still outside of the cell ("near-cell" method). The radial component of the initial velocity is converted into circular motion by the magnetic field. Because the orbit of the reduced cyclotron motion is tangent to the $z$ axis (axial symmetric axis of the cell) for an ion formed along the $z$ axis the maximum radial distance between the ion and the $z$-axis is two times the radius of the reduced cyclotron motion [11]. As an example, an aperture diameter of $2 \mathrm{~mm}$ and ions of $\mathrm{m} / q=5730$ $\mathrm{u} / \mathrm{e}$ in a 7-T field are considered: all ions with a higher initial radial velocity than only $60 \mathrm{~m} / \mathrm{s}$ collide with the front electrode (maximum radial distance is $1 \mathrm{~mm}$ at $60 \mathrm{~m} / \mathrm{s}$ ). Because the ions start at the $z$ axis and move toward the aperture with their initial axial velocities, the radial distance may be smaller at the aperture than the maximum radial distance that occurred the first time inside the cell. Because of this, the true limit of the initial radial velocity to pass through the aperture depends also on the initial axial velocity and the distance between the target and the aperture. Thereby the limit is generally somewhat higher for small distances than in the preceding example. However, in contrast to the in-cell method presented here, the aperture limits the number of trapped ion with an initial radial velocity. By using an external ionization method, the number of the trapped ions depends on the conversion of the initial radial velocity into axial velocity outside of the magnetic field and the transmission efficiency.

Because the number of the trapped ions is large with the in-cell method, the sensitivity must be high. Figure 9 shows a single scan spectra of gramicidin D 


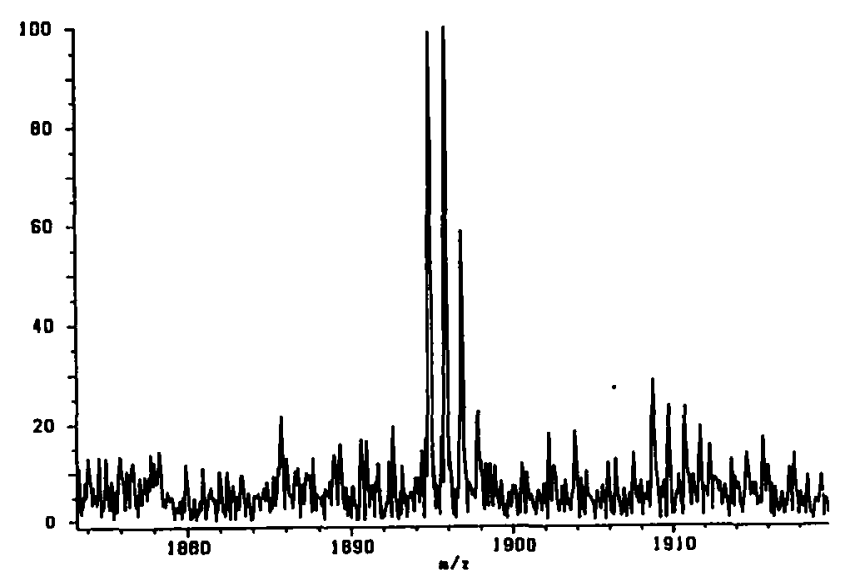

Figure 9. Spectrum with 9500 resolution on gramicidin D obtained after a single scan that consumed less than $1 \mathrm{fmol}$. A 100-fmol quantity of the analyte was applied to the target (analyte-DHB-fructose ratio was 1:3,000,000:0). More than 100 spectra comparable with the spectrum shown were possible with the applied sample.

obtained by consuming less than $1 \mathrm{fmol}$. A $0.1-\mu \mathrm{L}$ quantity of a $10^{-6}: 3-\mathrm{M}$ solution of gramicidin D-DHB was applied to the target (100 fmol of analyte). Ten different spots achieved comparable results. Each spot could be used for more than 10 laser shots, so the consumed quantity is less than $1 \mathrm{fmol}$ per experiment. By using a solution of $10^{-7}: 3 \mathrm{M}$, more than 10 scans were required for an appropriate signal-to-noise ratio and no further increase of the sensitivity per laser shot was achieved.

A comparison of the sensitivity of less than 1 fmol presented here with the sensitivities achieved with other MALDI-FTICR-MS methods for the most part is not possible because other researchers reported only the applied amount of sample, which expands over a range of $1-100$ pmol for a typical $10^{-4}-\mathrm{M}$ sample solution. Huang et al. [26] calculated the consumed quantity in the same way as described here. For one $\mathrm{MS}^{4}$ experiment, after a single laser shot, $10 \mathrm{pmol}$ were consumed. The consumed quantity per laser shot is roughly estimated in the picomole and subpicomole range by McIver et al. [19] and Sheng et al. [29], respectively.

\section{Reproducibility and Accuracy}

Although the trapping of ions with a wide range of initial velocities clearly increases the sensitivity, the resulting wide spread of radii of the circular motions prior to dipolar excitation is less favorable. Ions with high initial radii of motion are ejected during dipolar excitation. The optimal conditions for reproducibility and mass accuracy are a stable number of trapped ions and a negligible initial radius of motion. The first condition depends on the reproducibility of the MALDI technique. To reduce large radii the application of axialization is necessary to take full advantage of the high sensitivity of the in-cell method.
The mass difference between two different experiments with bovine insulin was $\sim 20 \mathrm{ppm}$ for the results presented here. No experiments for the calibration of the instrument were done. The absolute mass error, determined by a calibration for a $m / q$ range of $140-5740 \mathrm{u} / \mathrm{e}$ with data achieved partly under different conditions, is about $200 \mathrm{ppm}$. This error corresponds to results of Castoro and Wilkins [23] with a mass accuracy of 50-500 ppm, although they trapped the ions in the analyzer cell of a dual cell so that most of the ions with even small initial radial velocities are not trapped (long distance between target and conductance limit). Pastor et al. [25] improved the accuracy by a factor of $\sim 10$ by using axialization. High mass accuracy of masses higher than $3 \mathrm{ku}$ also were presented by $\mathrm{Li}$ et al. [20], who used an external source and pulsed collision gas but no quadrupolar excitation. The mass accuracy by the in-cell method should be increased also by axialization but without any restriction of the high sensitivity.

\section{Conclusion and Future Experiments}

These initial results show the potential of the new in-cell MALDI-FTICR-MS. The target as a part of one trapping electrode leads to a very efficient ion trapping. Spectra with resolved isotope peaks of up to $m / z=2$ ku can be obtained after single scans by using only the current customary FTICR-MS technique. The application of just a pulsed retarding potential increases the mass-to-charge ratio range to $5.7 \mathrm{ku}$ (16K resolution on bovine insulin after single scan). Ion storage times above $15 \mathrm{~s}$ are possible, although currently only for low resolution experiments. Very carefully balanced trapping voltages and-if needed-retarding voltage and retarding time are essential for long storage times or high resolution experiments. However, trapping for low resolution experiments is easy to achieve.

Whereas the ion trapping is very efficient but the ion storage is moderate, the application of quadrupolar excitation will improve the in-cell MALDI Fourier transform mass spectrometry method. The storage time, the mass resolution, and the mass accuracy can be increased by quadrupolar excitation $[13,25,27]$. The sensitivity expands by remeasurements that use quadrupolar excitation [44]. The trapping grids of the ICR cell described here allow fast pressure change and pumping. The eight side plates permit quadrupolar excitation to switch between quadrupolar excitation and dipolar excitation or image current detection without a relay circuit, which is usually required by single cell quadrupolar excitation experiments $[44,45]$. Two pairs of opposed electrodes operate for quadrupolar excitation and the other pairs of opposed plates can be utilized for dipolar excitation and for image current detection. Moreover, by application of the recently introduced quadrupolar excitation with only two op- 
posite plates [46], a cylindrical cell with six side plates would be sufficient for dipolar excitation, detection, and quadrupolar excitation without switching.

\section{Acknowledgments}

The authors thank F. H. Laukien (Bruker Analytical Systems Inc., Billerica, MA) and G. Baykut (Bruker-Franzen Analytik GmbH, Bremen, Germany) for the laser and optical components, and $C$. Köster (Bruker-Franzen Analytik GmbH) for helpful suggestions.

\section{References}

1. Karas, M.; Bachmann, D.; Bahr, U.; Hillenkamp, F. Int. J. Mass Spectrom. Ion Processes 1987, 78, 53-68.

2. Hillenkamp, F.; Karas, M.; Beavis, R. C.; Chait, B. T. Anal. Chem. 1991, 63, 1193A-1202A.

3. Wanczek, K. P. Int. J. Mass Spectrom. Ion Processes 1989, 95, 1-38.

4. Marshall, A. G.; Grosshans, P. B. Anal. Chem. 1991, 63, 215A-229A

5. Marshall, A. G.; Schweikhard, L. Int. J. Mass Spectrom. Ion Processes 1992, 118/119, 37-70.

6. Köster, C.; Kahr, M. S.; Castoro, J. A.; Wilkins, C. L. Mass Spectrom. Rev. 1992, 11, 495-512.

7. Buchanan, M. V.; Hettich, R. L. Anal. Chem. 1993, 65, 245A-259A.

8. Holliman, C. L.; Rempel, D. L.; Gross, M. L. Mass Spectrom. Rev. 1994, 13, 105-132.

9. Beavis, R. C.; Chait, B. T. Chem. Phys. Lett. 1991, 181, 479-484.

10. Hettich, R. L.; Buchanan, M. V. J. Am. Soc. Mass Spectrom. 1991, 2, 22-28.

11. Wood, T. D.; Schweikhard, L.; Marshall, A. G. Anal. Chem. 1992, 64, 1461-1469.

12. Dunbar, R. C.; Chen, J. H.; Hays, J. D. Int. J. Mass Spectrom. Ion Processes 1984, 57, 39-56.

13. Schweikhard, L.; Guan, S.; Marshall, A. G. Int. J. Mass Spectrom. Ion Processes 1992, 120, 71-83.

14. Holliman, C. L.; Rempel, D. L.; Gross, M. L. J. Am. Soc. Mass Spectrom. 1992, 3, 460-463.

15. Jeffries, J. B.; Barlow, S. E.; Dunn, G. H. Int. J. Mass Spectrom. Ion Processes 1983, 54, 169-187.

16. Rempel, D. L.; Huang, S. K.; Gross, M. L. Int. I. Mass Spectrom. Ion Processes 1986, 70, 163-184.

17. Caravatti, P.; Watson, C.; Knobeler, M.; Evard, D.; Laukien, F.; Wanczek, K. P. Proceedings of the 11th Informal Meeting on Mass Spectrometry; Budapest, Hungary, 1993; p 24.

18. McIver R. T.; Li, Y.; Hunter, R. L., Int. J. Mass Spectrom. Ion Processes 1994, 132, L1-L7.

19. McIver, R. T.; Li, Y.; Hunter, R. Proc. Natl. Acad. Sci. USA 1994, 91, 4801-4805.

20. Li, Y.; McIver, R. T.; Hunter, R. L. Anal. Chem. 1994, 66, 2077-2083.
21. Castoro, J. A.; Köster, C.; Wilkins, C. L. Rapid Commun. Mass Spectrom., 1992, 6, 239-241.

22. Köster, C.; Castoro, J. A.; Wilkins, C. L. J. Am. Chem. Soc. 1992, 114, 7572-7574.

23. Castoro, J. A.; Wilkins, C. L. Anal. Chem. 1993, 65, 2621-2627.

24. Castoro, J. A.; Köster, C.; Wilkins, C. L. Anal. Chem. 1993, 65, 784-788.

25. Pastor, S. J.; Castoro, J. A.; Wilkins, C. L. Anal. Chem. 1995, 67, 379-384.

26. Huang, Y.; Pasa-Tolic, L.; Guan, S.; Marshall, A. G. Anal. Chem. 1994, 66, 4385-4389.

27. Pasa-Tolic, L.; Huang, Y.; Guan, S.; Kim, H. S.; Marshall, A. G. J. Mass Spectrom. 1995, 30, 825-833.

28. Stemmler, E. A.; Hettich, R. L.; Hurst, G. B.; Buchanan, M. V. Rapid Commun. Mass Spectrom. 1993, 7, 828-836.

29. Sheng, L-S.; Covey, J. E.; Shew, S. L.; Winger, B. E.; Campana, J. E. Rapid Commun. Mass Spectrom. 1994, 8, 498-500.

30. Solouki, T.; Russell, D. H. Proc. Natl. Acad. Sci. USA 1992, 89, 5701-5704.

31. Anderson, J. S.; Harper, C. J.; Schmidt, E. G.; Laude, D. A. Proceedings of the 42nd ASMS Conference on Mass Spectrometry and Allied Topics, Chicago, IL, 1994; $\mathrm{p} 244$.

32. Kofel, P.; Allemann, M.; Kellerhals, Hp.; Wanczek, K. P. Int. J. Mass Spectrom. Ion Processes, 1985, 65, 97-103.

33. Kofel, P. Dissertation, Universität Bremen, 1987.

34. Hofstadler, S. A.; Laude, D. A. Int. J. Mass Spectrom. Ion Processes 1990, 101, 65-78.

35. Wang, M.; Marshall, A. G. Anal. Chem. 1989, 61, 1288-1293.

36. Bamberg, M.; Wanczek, K. P. Proceedings of the 37th ASMS Conference on Mass Spectrometry and Allid Topics; Miami Beach, FL, 1989; p 456.

37. Solouki, T.; Gillig, K. J.; Russell, D. H. Anal. Chem. 1994, 66. 1583-1587.

38. Savard, G.; Becker, S.; Bollen, G.; Kluge, H. J.; Moore, R. B.; Otto, T.; Schweikhard, L.; Stolzenberg, H.; Weiss, U. Phys. Lett. A 1991, 158, 247-252.

39. Guan, S.; Marshall, A. G. J. Chem. Phys. 1993, 98, 4486-4493.

40. Pezsa, I.; Knoll, H.; Wanczek, K. P.; Linden H. B. Proceedings of the 36th ASMS Conference on Mass Spectrometry and Allied Topics, San Francisco, CA, 1988; $\mathrm{p} 618$.

41. Anderson, J. S.; Laude, D. A.; Proceedings of the 43rd ASMS Conference on Mass Spectrometry and Allied Topics; Atlanta, GA, 1995, p 793.

42. Williams, E. R.; Henry, K. D.; McLafferty, F. W. J. Am. Chem. Soc. 1990, 112, 6157-6162.

43. Guan, Z.; Hofstadler, S. A.; Laude, D. A. Anal. Chem. 1993, $65,1588-1593$.

44. Speir, J. P.; Gorman, G. S.; Pitsenberger, C. C.; Turner, C. A.; Wang, P. P.; Amster I. J. Anal. Chem. 1993, 65, 1746-1752.

45. Bruce, J. E.; Anderson, G. A.; Hofstadler, S. A.; Van Orden, S. L.; Sherman, M. S.; Rockwood, A. L.; Smith, R. D. Rapid Commun. Mass Spectrom. 1993, 7, 914-919.

46. Hendrickson, C. L.; Drader, J. J.; Laude, D. A. J. Am. Soc. Mass Spectrom. 1995, 6, 448-452. 